Another reason why adults find it hard to draw accurately

\author{
W.J. Matthews
}

University of Warwick

\author{
Amy Adams
}

University of Leicester

WORD COUNT: 1471

ABSTRACT WORD COUNT: 117

Address correspondence to:

William Matthews

Department of Psychology,

University of Warwick,

Coventry

CV4 7AL

UK

Tel: +44/0 2476523096

Fax: +44/0 2476524225

Email:w.j.matthews@warwick.ac.uk 
Matthews \& Adams

\begin{abstract}
Misperception of objects is a major cause of inaccuracies in adults' drawing. It has previously been established that participants' drawings are biased by their knowledge of the drawn object. We hypothesized that additional inaccuracy arises because drawings are biased towards participants' idiosyncratic canonical representations of the object. We report that participants' free drawings of a cylinder are correlated with their observational drawings of the same shape, providing evidence that people's observational drawings are distorted by their individual schematic representations of the objects in question. It is unclear whether this reflects a perceptual distortion or a bias in drawing production; in either case, this result provides a further explanation for why people are poor at drawing from observation.
\end{abstract}




\section{Another reason why adults find it hard to draw accurately}

Why do most people find it so difficult to make observational drawings? Cohen \& Bennett (1997) established that the primary source of errors in adults' observational drawings is misperception of the object. A major contributor to this misperception is participants' knowledge about the properties of the object they are drawing. For example, Taylor \& Mitchell (1997) established that adults' knowledge contaminates their perceptions. These authors presented participants with an illuminated disc in a dark chamber devoid of perspective cubes, and asked them to match a computer generated ellipse to the model. Half of the participants were shown at the start of the experiment that the disc was in fact circular; half were not. The former group exaggerated the circularity of the ellipse while the latter group did not, providing evidence that knowledge of the stimulus' true shape contaminated judgments of its appearance. In a more recent study, Mitchell, Ropar, Ackroyd, \& Rajendran (2005) demonstrated that such misperceptions lead to drawing errors. Thus adults, like children, seem to "draw what they know" rather than what they see.

Work such as this strongly suggests that adults' drawings are inaccurate because they misperceive the model object, and that this misperception arises partly because of knowledge about the "true" form of the shape they are 
Matthews \& Adams

drawing. However, these studies have investigated the extent to which everyone's drawings are biased towards the same internal, knowledge-based representation - e.g. a circle. Yet it may also be that each person has his or her own internal, schematic representation of a given object, and that each participant's observational drawing is pulled towards this unique canonical representation. (Note that we, like e.g. Picard \& Durand (2005), use the term canonical to refer to an internal representation of an object or shape which can differ between individuals).

We can seek to establish an individual's canonical representation by asking him or her to produce a free drawing, a technique employed by many researchers investigating children's drawings including, recently, Picard \& Durand (2005). These authors asked 4-6 year old children to produce a free drawing of a saucepan before attempting observational drawings of a 3D saucepan with its handle in different orientations. They found that, although not the only source of error, the youngest children showed a tendency to draw the handle in the same orientation as in their free drawings. This canonical bias diminished with age, but we hypothesized that, with a more subtle test, adults might reveal the same tendency to bias their observational drawings towards their (individually varying) canonical representations of the object. 
Matthews \& Adams

To test this possibility, we asked 72 participants to produce a line drawing of a cylinder based upon their mental representations of this shape. They then spent approximately ten minutes completing a separate experiment, before being asked to draw a (previously concealed) cylinder from observation. The cylinder stood upright on a table and was $26 \mathrm{~cm}$ high and $8 \mathrm{~cm}$ in diameter. Participants used a chin-rest set $82 \mathrm{~cm}$ from the base of the cylinder; eye-level was $41 \mathrm{~cm}$ above the table.

One participant was discarded because her free drawing was of a cuboid. For the remaining 142 drawings both authors independently measured the lengths labelled A, B, C and D in Figure 1.

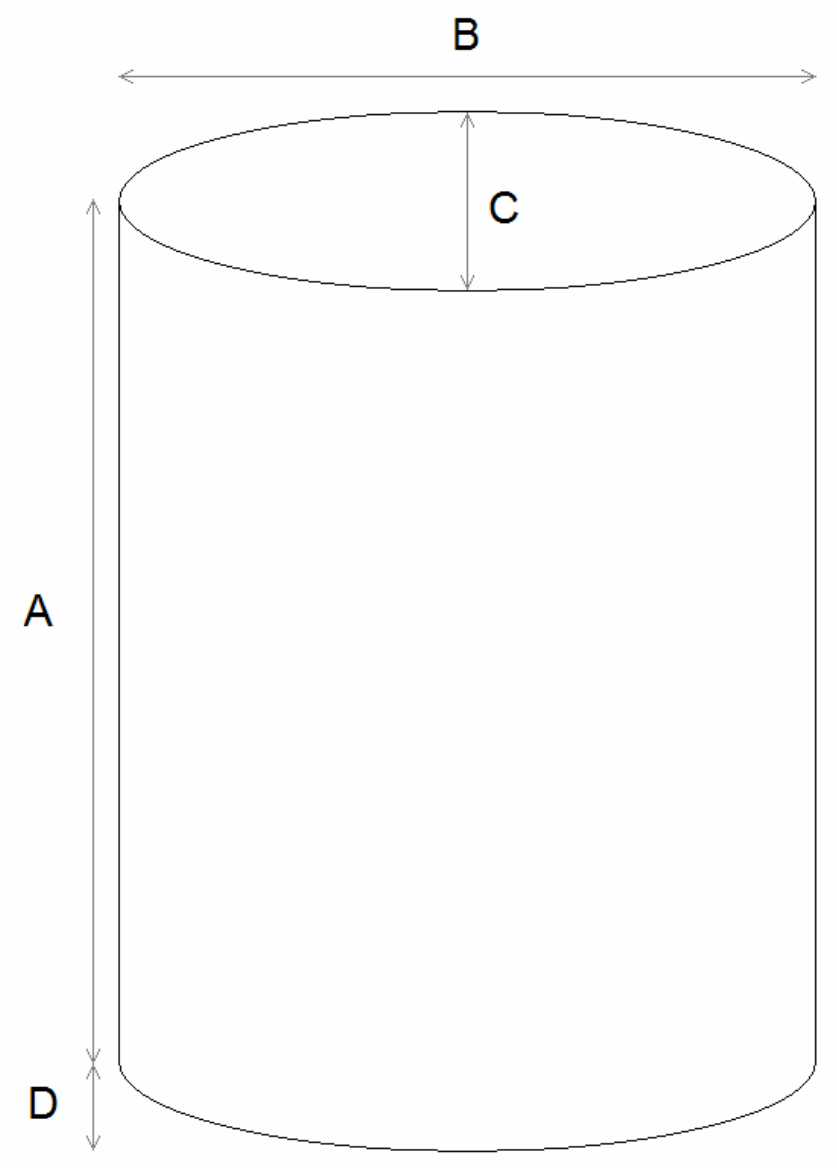


Matthews \& Adams

Figure 1. The measurements taken from each drawing. Length A was measured for both left and right sides, and an average taken.

Inter-rater agreement was excellent $\left(R^{2}>0.98\right)$ and the two measurements for each length were averaged. To control for individual differences in the absolute size of the shapes drawn, we focussed on the ratios of the lengths measured (Mitchell et al, 2005). There were six ratios of interest: $\mathrm{A} / \mathrm{B}, \mathrm{A} / \mathrm{C}, \mathrm{A} / \mathrm{D}, \mathrm{B} / \mathrm{C}, \mathrm{B} / \mathrm{D}$ and $\mathrm{C} / \mathrm{D}$. Values were log-transformed to improve normality. We performed correlation analysis to see whether the ratios that participants used in their free drawings predicted the corresponding ratios in their observational drawings. Participants' free drawings fell into two groups: those where the cylinder was upright with the ellipse at the top $(N=48)$, and those where the cylinder was on its side, with the ellipse at the front $(N=23)$. Table 1 shows the results for both groups separately, and when group is ignored. 


\begin{tabular}{|c|c|c|c|c|c|c|c|}
\hline & & $\mathrm{A} / \mathrm{B}$ & $\mathrm{A} / \mathrm{C}$ & $\mathrm{A} / \mathrm{D}$ & $\mathrm{B} / \mathrm{C}$ & $\mathrm{B} / \mathrm{D}$ & $\mathrm{C} / \mathrm{D}$ \\
\hline \multirow{2}{*}{ Upright } & $r$ & .336 & .300 & .434 & .255 & .458 & .275 \\
\hline & $p$ & .020 & .038 & .003 & .080 & .001 & .065 \\
\hline \multirow{2}{*}{ Side } & $r$ & .277 & .212 & .110 & .059 & .104 & .279 \\
\hline & $p$ & .200 & .332 & .618 & .790 & .319 & .198 \\
\hline \multirow{2}{*}{ Collapsed } & $r$ & .352 & .176 & .321 & .112 & .421 & .144 \\
\hline & $p$ & .003 & .141 & .007 & .351 & .001 & .239 \\
\hline
\end{tabular}

Table 1. Correlations between ratios in free drawings and the corresponding ratios in the observational drawings. Values are Pearson's $r$ with corresponding $p$-value (two-tailed). Two participants produced flat lines at the base of their drawings (i.e. D was zero); ratios involving D could not be computed for these participants and they were excluded from these correlations.

All of the examined correlations are positive; when canonical orientation is ignored, three of the six correlations are highly significant and, in the case of the participants whose free drawings were upright, four are significant and the remaining two approach significance. This provides strong evidence that participants' observational drawings are biased towards their idiosyncratic canonical representations of the shape. We emphasize that this result is very different from previous findings showing that drawings are contaminated by knowledge of object properties. All participants may make 
Matthews \& Adams

their drawing of the top surface of the cylinder overly circular because they know the geometric properties of the model shape. Indeed, comparison of the ratio $\mathrm{B} / \mathrm{C}$ in the observational drawings with the ratio in a photograph taken from the participants' position showed that people significantly "circularized" their drawings. However, the shape towards which the observational drawing is biased must be the same for everyone (since presumably everyone knows that a cylinder has circular ends). As such, this factor cannot explain the correlations between free drawings and observational drawings that we found. Moreover, some of the ratios correlated between the canonical and observational drawings, such as the height-to-width ratio $(\mathrm{A} / \mathrm{B})$, do not have any obvious connection with knowing about object properties. Irrespective of any tendency to make the drawing look like what they know about the model cylinder, participants make their observational drawings look like their individual canonical representations.

It is noticeable that none of the correlations were significant for participants whose canonical cylinders were lying on their side. This may be because of low statistical power: there were only 23 people in this group, and their drawings were relatively similar. However, it may also be because their canonical representations involved a different viewpoint from that of the upright model cylinder, an intriguing possibility which merits future study. It is 
Matthews \& Adams

also noticeable that, when canonical orientation is ignored, none of the significant correlations involve $\mathrm{C}$ (the depth of the ellipse). One possible explanation for this is that, since this dimension is particularly sensitive to knowledge of the object's "true" shape, there is less inter-individual variation in participants' canonical representations of this aspect of the stimulus. Again, this possibility is amenable to future investigation.

One limitation of the current result is that it is not clear whether the correlation between free and observational drawings reflects a bias of perception or of production. It may be that each participant's perception of the model cylinder is biased towards his or her idiosyncratic canonical representation (or towards the memory of the recently produced free drawing). Alternatively, the free drawings may represent each participants' understanding of how such a shape should be drawn; when they make their observational drawings, they make use of the same production schema. Disentangling these possibilities will be a useful direction for future research. In either case, it seems that part of the reason why adults struggle to produce accurate drawings is because they are biased towards using certain proportions which are specific to the individual and independent of both the appearance of the object in front of them and their knowledge of that object. 
Matthews \& Adams

\section{Acknowledgements}

We thank Neil Stewart and Luciano Buratto for useful discussion, and

Elizabeth Sheppard for helpful comments on an earlier version of this article. 
Matthews \& Adams

\author{
References
}

Cohen D J, Bennett S 1997 "Why can't most people draw what they see?" Journal of Experimental Psychology: Human Perception and Performance 23 609-621

Mitchell P, Ropar D, Ackroyd K, Rajendran G 2005 “How perception impacts on drawings" Journal of Experimental Psychology: Human Perception and Performance 31 996-1003

Picard D, Durand K 2005 “Are young children’s drawings canonically biased?" Journal of Experimental Child Psychology 90 48-64

Taylor L M, Mitchell P 1997 “Judgments of apparent shape contaminated by knowledge of reality: Viewing circles obliquely" British Journal of Psychology 88 653-670 